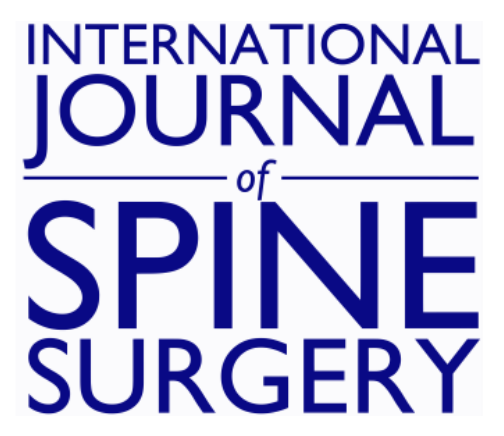

\title{
Fenestrated Pedicle Screws in Spinal Oncology: Technique and Comparative Retrospective Analysis
}

Saavan Patel, Rown Parola, Clayton L. Rosinski, Ravi S. Nunna and Ankit I. Mehta

Int J Spine Surg 2021, 15 (1) 113-118

doi: https://doi.org/10.14444/8015

http://ijssurgery.com/content/15/1/113

This information is current as of April 26, 2023.

Email Alerts Receive free email-alerts when new articles cite this article. Sign up at:

http://ijssurgery.com/alerts

The International Journal of Spine Surgery

2397 Waterbury Circle, Suite 1,

Aurora, IL 60504, Phone: +1-630-375-1432 


\title{
Fenestrated Pedicle Screws in Spinal Oncology: Technique and Comparative Retrospective Analysis
}

\author{
SAAVAN PATEL, BS, ROWN PAROLA, MS, CLAYTON L. ROSINSKI, BS, RAVI S. NUNNA, MD, \\ ANKIT I. MEHTA, MD \\ Department of Neurosurgery, University of Illinois at Chicago, Chicago, Illinois
}

\begin{abstract}
Background: The use of spinal stabilization with decompression has been shown to improve survival, spinal stability, and ambulatory status in patients with metastatic spinal tumors. However, the poor bone quality typically seen in these patients can prevent adequate stabilization. Fenestrated pedicle screws permit augmented fixation via injection of bone cement into the vertebral body upon screw placement, potentially mitigating the difficulties in achieving adequate stabilization in these patients.

Objective: To compare surgical outcomes of posterior spinal fusion in patients with cancerous spinal lesions between polymethyl methacrylate cement-augmented fenestrated screws and standard pedicle screws.

Methods: A total of 19 consecutive patients with cancerous spinal lesions receiving posterior spinal fusion (PSF) with pedicle screws from a single surgeon were retrospectively reviewed for demographic information, comorbidities, surgical parameters, and outcomes.

Results: Ten patients underwent PSF with cement augmentation, whereas 9 underwent standard PSF. There was no significant difference in demographics, comorbidities, or surgical characteristics. Operative time was significantly greater in the cement-augmented group (302 \pm 100 minutes vs $203 \pm 55$ minutes; $P=.015)$. There was no significant difference in rates of operation or readmission between the cohorts nor was there any significant difference in discharge disposition. There was 1 case of surgical site infection (in a patient with a fenestrated screw) and no cases of cement extravasation. No instances of mechanical hardware failure were recorded.

Conclusions: Fenestrated screws confer similar risk profiles as nonfenestrated screws for posterior spinal fusion in patients with spinal cancer. However, fenestrated screws may affect operative time, radiation exposure, and impose risk of cement extravasation. Cement-augmented fenestrated pedicle screws may be a viable option for patients with poor bone quality associated with metastatic disease without significantly increased rates of surgical complications.
\end{abstract}

Level of Evidence: 3.

Tumor

Keywords: fenestrated, screws, cement, augmentation

\section{INTRODUCTION}

In the management of metastatic spinal tumors, the use of spinal stabilization with decompression has been shown to improve survival, spinal stability, and ambulatory status in patients. ${ }^{1}$ Separation surgery is commonly used to achieve maximal circumferential epidural decompression, posterior spine stabilization, and separation of the tumor from neural elements such that adjuvant radiosurgery can achieve adequate tumor control. ${ }^{2-4}$ However, poor bone quality resulting from the oncological process and osteolysis often compromises stability of the surgical construct. A major cause of morbidity with posterior fusion is mechanical failure due to screw loosening. ${ }^{5}$ Recently, pedicle screws have been developed with a central cannula and fenestrations that allow for cement augmentation, providing another potential solution to screw loosening and backing out, particularly in patients with poor bone quality. ${ }^{5-7}$

Whereas fenestrated pedicle screws augmented with cement have demonstrated an increase in purchase and pullout strength in biomechanical studies, ${ }^{8-10}$ they also confer an additional risk of cement extravasation. This study sought to offer a technique in addition to comparing efficacy, clinical outcomes, and complications in patients with spinal tumors undergoing posterior spinal fusion with cement-augmented fenestrated pedicle screws versus nonfenestrated pedicle screws. 


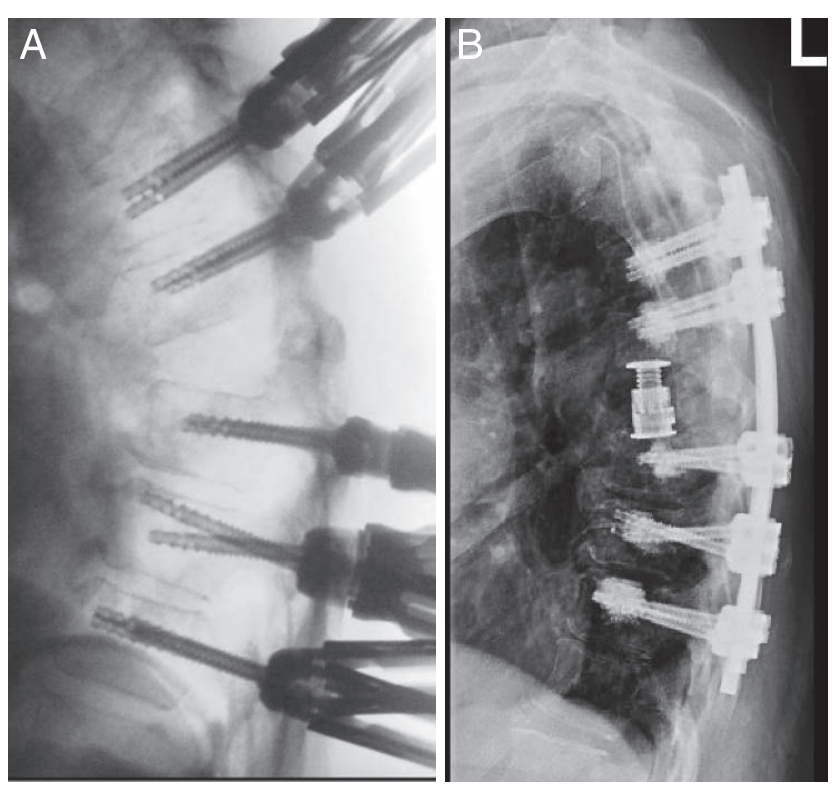

Figure. Radiographic demonstration of fenestrated screw implantation and subsequent cement augmentation. (Left) Lateral radiograph of implanted fenestrated pedicle screws prior to cement augmentation. (Right) Lateral radiograph of implanted fenestrated pedicle screws after cement augmentation with cement visible at distal end of screw.

\section{METHODS}

Eligibility for this study was limited to adult patients with primary or metastatic spine lesions who received posterior spinal fusion with pedicle screws from a single surgeon at a tertiary academic center between January 2016 and March 2018. A total of 19 consecutive patients were identified for this retrospective review, which was approved by the institutional review board. Patient consent for participation was not required due to the retrospective nature of the study. Patients received fenestrated or nonfenestrated screws depending on their surgery date, with patients with operations after March 2017 receiving fenestrated pedicle screws and patients with operations February 2017 or earlier receiving nonfenestrated pedicle screws. After obtaining approval from the institutional review board, the electronic medical records of identified patients were used to collect demographic information (sex, age at time of surgery), comorbidities (hypertension, smoking status, obesity, diabetes mellitus), surgical parameters (previous surgical intervention, number of levels receiving laminectomy, additional corpectomy), and outcomes (readmission, reoperation, length of stay, surgery duration, blood loss, surgical site infection, and discharge destination). Postsurgical data were col- lected for all patients to October 2018 when available.

Data collection and analysis was performed in $\mathrm{R}$, version 3.5.1 (R Foundation, Vienna, Austria) using an unpaired $t$ test for discrete variables and Pearson $\chi^{2}$ test for categorical variables. Missing follow-up data were addressed using intent-to-treat analysis whereby unreported postoperative infections, readmission, or reoperation were analyzed as though they had not occurred.

Cement augmentation is accomplished by injection of polymethyl methacrylate (PMMA) through a central cannulation of the screw that terminates in 3 or 6 fenestrations in the distal portion of the thread where the PMMA is extruded into the vertebral body. In the insertion of fenestrated screws, pedicles may be targeted in the same manner as any nonfenestrated screw; this can be performed under fluoroscopic guidance, navigation, or freehand. However, extra care should be taken during screw measurement and placement to avoid a breach of the cortical surface at the distal end (Figure [left]). Upon screw insertion, we suggest use of intraoperative 2-dimensional or 3-dimensional imaging (O-arm surgical imaging system, Medtronic, Dublin, Ireland) to confirm screw placement and ensure there is no breach of the pedicle wall or anterior cortex of the vertebral body. This is especially important in the use of fenestrated screws for cement delivery to avoid cement extrusion into the retroperitoneal space or spinal canal.

Once adequate screw placement has been confirmed, a cement delivery system can be prepared. At this point, intraoperative fluoroscopy should be used such that the radiopaque bone-cement injection can be done under continuous radiological visualization. Before bone-cement preparation, the alignment guiding system must be assembled on every inserted screw intended for cement augmentation to prevent undue stress on the cannula and subsequent cannula breakage. Bone cement can then be prepared and loaded into its reservoir, which can then be connected to flexible tubing attached to the hand pump. This pump-reservoir apparatus can then be connected to the open cannula to attach the entire system to the assembled alignment guides.

At this point, bone cement can begin to be delivered. Controlled cement delivery with slow rotation of the hand pump and direct fluoroscopic visualization is essential to achieve satisfactory 
Table 1. Comparison of demographics, comorbidities, and surgical characteristics by cohort.

\begin{tabular}{|c|c|c|c|c|}
\hline & Total & Fenestrated & Nonfenestrated & $P$ Value $^{a}$ \\
\hline Total patients, n (\%) & $19(100)$ & $10(52.6)$ & $9(47.4)$ & .13 \\
\hline Male, n (\%) & $11(57.9)$ & $3(30.0)$ & $5(55.6)$ & \\
\hline Female, n $(\%)$ & $8(42.1)$ & $7(70.0)$ & $4(44.4)$ & \\
\hline Age, mean $\pm \mathrm{SD}, \mathrm{y}$ & $61.8 \pm 9.6$ & $58.9 \pm 10.2$ & $65 \pm 8.3$ & .16 \\
\hline Hypertension, n (\%) & & & & 1.00 \\
\hline Yes & $14(73.7)$ & $7(70.0)$ & $7(77.8)$ & \\
\hline No & $5(26.3)$ & $3(30.0)$ & $2(22.2)$ & \\
\hline Smoker, n $(\%)$ & & & & .26 \\
\hline Yes & $10(52.6)$ & $7(70.0)$ & $3(33.3)$ & \\
\hline No & $9(47.4)$ & $3(30.0)$ & $6(66.7)$ & \\
\hline Obesity, n (\%) & & & & .52 \\
\hline Yes & $6(31.6)$ & $2(20.0)$ & $4(44.4)$ & \\
\hline No & $13(68.4)$ & $8(80.0)$ & $5(55.6)$ & \\
\hline Diabetes mellitus, n (\%) & & & & 1.00 \\
\hline Yes & $4(21.1)$ & $2(20.0)$ & $2(22.2)$ & \\
\hline No & $15(78.9)$ & $8(80.0)$ & $7(77.8)$ & \\
\hline Previous surgical intervention, n (\%) & & & & .25 \\
\hline Yes & $3(15.8)$ & $3(30.0)$ & $0(0.0)$ & \\
\hline No & $16(84.2)$ & $7(70.0)$ & $9(100.0)$ & \\
\hline $\begin{array}{l}\text { Number of levels receiving } \\
\text { laminectomy, mean } \pm \mathrm{SD}\end{array}$ & $2.6 \pm 1.8$ & $2.3 \pm 2.3$ & $2.9 \pm 1.1$ & .48 \\
\hline Corpectomy, n (\%) & & & & .66 \\
\hline Yes & $4(21.1)$ & $3(30.0)$ & $1(11.1)$ & \\
\hline No & $15(78.9)$ & $7(70.0)$ & $8(88.9)$ & \\
\hline \multicolumn{5}{|l|}{ Type of metastasis, n (\%) } \\
\hline Lytic & $13(68.4)$ & $9(90.0)$ & $4(44.4)$ & \\
\hline Blastic & $2(10.5)$ & $1(10.0)$ & $1(11.1)$ & \\
\hline Mixed & $2(10.5)$ & $0(0.0)$ & $2(22.2)$ & \\
\hline Extrinsic & $2(10.5)$ & $0(0.0)$ & $2(22.2)$ & \\
\hline
\end{tabular}

${ }^{\text {a}}$ Statistically significant: $P<.05$.

results and prevent cement leakage or extravasation. Once an appropriate amount of cement has been introduced, delivery can be stopped and the cannula can be disengaged from the alignment guide. An appropriate amount of cement may be considered to create a small, well-circumscribed cloud at the distal end of the screw (Figure [right]). This procedure is repeated for each screw intended for cement augmentation. Upon completion of cement introduction into all screws, alignment guides can be removed from each screw head, being careful to avoid any torsional movements because cement is setting during this time. Once all alignment guides are removed, appropriately sized and contoured rods can be placed into the polyaxial screw heads and captured with placement of set screws. Once cement setting time has lapsed (10-15 minutes depending on temperature), set screws can be final tightened, thus completing cement-augmented spinal fusion.

\section{RESULTS}

Participants included 10 patients who received posterior spinal fusion with fenestrated pedicle screws and cement-augmented screws and 9 patients who received posterior spinal fusion with non- fenestrated pedicle screws. All patients identified had metastatic spinal lesions. Of the 10 patients who received posterior spinal fusion with fenestrated pedicle screws, 9 had lytic metastases, compared with 4 of the 9 patients who received posterior spinal fusion with nonfenestrated pedicle screws. Participants included 11 men and 8 women with a mean age of 61.8 years (Table 1). The 2 groups did not significantly differ along demographic parameters including presentation with primary versus metastatic spinal lesion, any measured comorbidities, previous surgical intervention, number of levels receiving laminectomy, or additional corpectomy.

Of all measured outcomes, just surgery duration differed significantly between patients receiving posterior fixation with nonfenestrated and those receiving fenestrated pedicle screws (Table 2). On average, surgery duration for the fenestrated group was significantly longer than for the nonfenestrated group ( $302 \pm 100$ minutes vs $203 \pm 55$ minutes; $P=$ .015). It is important to note that this difference existed despite the number of levels operated upon and the frequency of corpectomy being performed not differing significantly between the groups. Blood loss trended towards being greater in patients with fenestrated screws as well $(920 \pm 579$ vs $489 \pm 434$; 
Table 2. Comparison of outcomes by cohort.

\begin{tabular}{|c|c|c|c|c|}
\hline & Total $(\mathrm{N}=19)$ & Fenestrated $(\mathrm{n}=10)$ & Nonfenestrated $(\mathrm{n}=9)$ & $P$ Value $^{\mathrm{a}}$ \\
\hline Readmissions, $\mathrm{n}(\%)$ & $3(15.8)$ & $1(10.0)$ & $2(22.2)$ & .92 \\
\hline Reoperation, $\mathrm{n}(\%)$ & $1(5.3)$ & $0(0.0)$ & $1(11.1)$ & .96 \\
\hline Length of stay, mean $\pm \mathrm{SD}, \mathrm{d}$ & $11.4 \pm 8.7$ & $13.5 \pm 11.6$ & $9 \pm 2.8$ & .26 \\
\hline Surgery duration, mean $\pm \mathrm{SD}$, min & $255.4 \pm 94.3$ & $302.6 \pm 99.5$ & $203 \pm 54.8$ & $.02^{\mathrm{a}}$ \\
\hline Blood loss, mean $\pm \mathrm{SD}, \mathrm{mL}$ & $716 \pm 548$ & $920 \pm 579$ & $489 \pm 434$ & .08 \\
\hline Surgical site infection, n (\%) & $1(5.3)$ & $1(10.0)$ & $0(0.0)$ & 1.00 \\
\hline Cement extravasation, $\mathrm{n}(\%)$ & $0(0.0)$ & $0(0.0)$ & $0(0.0)$ & \\
\hline Discharge disposition & & & & .12 \\
\hline Home, n (\%) & $7(36.8)$ & $2(20.0)$ & $5(55.6)$ & \\
\hline Acute rehab, n (\%) & $5(26.3)$ & $3(30.0)$ & $2(22.2)$ & \\
\hline Subacute rehab, n (\%) & $3(15.8)$ & $1(10.0)$ & $2(22.2)$ & \\
\hline Veterans Affairs, n (\%) & $4(21.1)$ & $4(40.0)$ & $0(0.0)$ & \\
\hline
\end{tabular}

${ }^{a}$ Statistically significant: $P<.05$.

$P=.08)$. Last, it should be noted that there was just 1 case of surgical site infection (in a fenestrated patient) and no cases of intraoperative cement extravasation or postoperative complications resulting from cement extravasation. There was no significant difference in discharge disposition or hospital length of stay between the groups.

Of the 90 fenestrated screws that were placed bilaterally in 45 vertebral bodies, only 1 was not augmented with PMMA. The nonaugmented screw was at the T7 level and corresponded to the increased risk for extravasation as pedicle size decreased at more superior levels. The screw was not augmented with PMMA due to increased risk of extravasation, which was based on intraoperative screw placement imaging.

Three patients were readmitted to the hospital following surgery. One instance of readmittance in the fenestrated group was due to terminal brain metastases. The other 2 instances of readmittance in the nonfenestrated group were due to hip pain from tumor burden 414 days postsurgery and sacrectomy for recurrent metastatic lesion 474 days postsurgery, respectively. No instances of mechanical hardware failure were recorded.

\section{DISCUSSION}

Several studies have described the use of fenestrated pedicle screws and characterized the surgical outcomes, ${ }^{5,6,10-14}$ but this is the first study comparing outcomes from a single surgeon using fenestrated and nonfenestrated pedicle screws for consecutive patients with primary or metastatic spine lesions in the United States. It is important to note that these 2 groups of patients did not differ significantly in any of the measured demographics, comorbidities, or surgical characteristics. In addition, the fact that these patients with cancerous spinal lesions were all operated upon sequentially by the same surgeon further facilitated comparison between posterior spinal fusion with nonfenestrated and cement-augmented fenestrated pedicle screws.

This series revealed similar findings between groups treated with nonfenestrated and fenestrated pedicle screws. The 2 groups notably differed in operative time, with significantly longer surgery durations for the fenestrated group. This difference persists when controlling for larger surgeries as determined by an increased number of vertebrae receiving laminectomy. Surgery-duration differences are attributed to differences in surgical technique. Use of either screw dictates the use of fluoroscopy to confirm proper screw placement. However, use of fenestrated screws with cement augmentation differs in that following confirmation of placement the PMMA must be prepared, loaded into the administration device, inserted down the central canal under continuous $\mathrm{x}$-ray fluoroscopy to ensure proper filling of the screw and extrusion into the vertebral body, and allowed to cure for 300 seconds. These additional steps added considerable time to the procedure, creating the observed difference in surgery duration. In addition, the increased length of surgery duration may be the reason that blood loss trended towards being significantly greater in the fenestrated screw group.

Increased surgery duration has previously been shown ${ }^{15,16}$ to increase the risk for surgical site infection (SSI). However, despite the significantly increased surgical duration in patients receiving fenestrated screws, the difference in infection rate did not differ significantly (Table 2). In fact, the overall SSI rate was very low, with just $1(5.3 \%)$ patient developing an SSI. The infection rate of $10 \%$ in the fenestrated group is similar to the SSI 
rates previously reported, ${ }^{5,6}$ which ranged from $8 \%-10 \%$.

The benefit of using cement augmentation with fenestrated pedicle screws is the added pullout strength and purchase, which has been repeatedly demonstrated. ${ }^{6,9,17}$ One risk of using cement augmentation with fenestrated pedicle screws is the potential for cement extravasation. In this comparison, there were no instances of cement extravasation, demonstrating that the risk may be minimized by being cautious with use of cement and ensuring that cement is inserted into only properly placed screws with O-arm confirmation. Rates of cement extravasation have been previously reported ${ }^{5,6,14}$ to be as high as $4 \%-25 \%$, although 1 study of 98 cases using fenestrated screws with cement augmentation revealed a rate of clinically relevant cement augmentation of $0 \% .{ }^{11}$ Whereas our study was unable to ascertain or compare long-term functional outcomes in these patients, we were able to demonstrate the ability of cement-augmented fenestrated pedicle screw fixation to achieve adequate fixation without significantly increased risk. Thus, in patients with metastatic spinal disease with notoriously poor bone quality, the use of cement augmentation has the potential to fortify spinal fixation without increasing surgical morbidity. The subsequent potential for stronger constructs could prevent hardware failure and thus spare these already severely ill patients from requiring reoperation and the associated morbidity and mortality. Ultimately, cement-augmented fenestrated pedicle screws may be a valuable option in patients with poor bone quality secondary to tumor due to the increased biomechanical strength and largely similar surgical complication profile compared with nonfenestrated screws.

The main limitation of the present study is the small number of patients included in the comparison. Thus, this analysis was not designed to draw conclusions; however, we believe comparing the 2 screw types in 2 similar patient groups helped facilitate some degree of comparison. The retrospective design of the experiment is also associated with some limitations. In addition, the follow-up time may not have been sufficient to fully assess mechanical differences between the 2 screw types. Nonetheless, the study is the first direct comparison of acute surgical outcomes between patients with neoplastic spinal lesions undergoing posterior fusion with either nonfenestrated or cement-augmented fenestrated pedicle screws, and we hope the technique assists providers in optimizing outcomes with fenestrated pedicle screws. Prudent future research would include prospective trials that seek to compare these 2 groups with an extensive followup period.

\section{CONCLUSION}

Fenestrated screws largely confer similar risk profiles as nonfenestrated screws for posterior spinal fusion in patients with spinal cancer. However, fenestrated screws may affect operative time and radiation exposure. Sequential fluoroscopic imaging during PMMA injection may add significantly to both operative time and radiation exposure. In addition, the risk of PMMA cement extravasation should also be considered. Cement-augmented fenestrated pedicle screws may be a viable option for patients with poor bone quality associated with metastatic disease without significantly increasing rates of surgical complications.

\section{REFERENCES}

1. Patchell RA, Tibbs PA, Regine WF, et al. Direct decompressive surgical resection in the treatment of spinal cord compression caused by metastatic cancer: a randomised trial. Lancet. 2005;366(9486):643-648. doi:10.1016/S01406736(05)66954-1

2. Bilsky MH, Laufer I, Burch S. Shifting paradigms in the treatment of metastatic spine disease. Spine. 2009;34(suppl):S101S107. doi:10.1097/BRS.0b013e3181bac4b2

3. Bate BG, Khan NR, Kimball BY, Gabrick K, Weaver J. Stereotactic radiosurgery for spinal metastases with or without separation surgery. J Neurosurg Spine. 2015;22(4):409-415. doi:10.3171/2014.10.SPINE14252

4. Barzilai O, Laufer I, Robin A, Xu R, Yamada Y, Bilsky MH. Hybrid therapy for metastatic epidural spinal cord compression: technique for separation surgery and spine radiosurgery. Oper Neurosurg. 2019;16(3):310-318. doi:10. 1093/ons/opy137

5. Amendola L, Gasbarrini A, Fosco M, et al. Fenestrated pedicle screws for cement-augmented purchase in patients with bone softening: a review of 21 cases. J Orthopaed Traumatol. 2011;12(4):193-199. doi:10.1007/s10195-011-0164-9

6. Abousayed M, Boktor JG, Sultan AM, Koptan W, ElMiligui Y. Augmentation of fenestrated pedicle screws with cement in patients with osteoporotic spine. J Craniovertebr Junction Spine. 2018;9(1):20-25. doi:10.4103/jcvjs.JCVJS_14_18

7. Singh V, Mahajan R, Das K, Chhabra HS, Rustagi T. Surgical trend analysis for use of cement augmented pedicle screws in osteoporosis of spine: a systematic review (2000-2017). Global Spine J. 2019;9(7):783-795. doi:10.1177/2192568218801570

8. Zindrick MR, Wiltse LL, Widell EH, et al. A biomechanical study of intrapeduncular screw fixation in the lumbosacral spine. Clin Orthop Relat Res. 1986;(203):99-112.

9. Paré PE, Chappuis JL, Rampersaud R, et al. Biomechanical 
evaluation of a novel fenestrated pedicle screw augmented with bone cement in osteoporotic spines. Spine. 2011;36(18):E1210 E1214. doi:10.1097/BRS.0b013e318205e3af

10. Moon BJ, Cho BY, Choi EY, Zhang HY. Polymethylmethacrylate-augmented screw fixation for stabilization of the osteoporotic spine: a three-year follow-up of 37 patients. $J$ Korean Neurosurg Soc. 2009;46(4):305-311. doi:10.3340/jkns. 2009.46.4.305

11. Mueller JU, Baldauf J, Marx S, Kirsch M, Schroeder HWS, Pillich DT. Cement leakage in pedicle screw augmentation: a prospective analysis of 98 patients and 474 augmented pedicle screws. J Neurosurg Spine. 2016;25(1):103-109. doi:10. 3171/2015.10.SPINE15511

12. Ulusoy OL, Kahraman S, Karalok I, et al. Pulmonary cement embolism following cement-augmented fenestrated pedicle screw fixation in adult spinal deformity patients with severe osteoporosis (analysis of 2978 fenestrated screws). Eur Spine J. 2018;27(9):2348-2356. doi:10.1007/s00586-018-5593-1

13. Ghermandi R, Pipola V, Colangeli S, et al. Polymethylmethacrylate-augmented fenestrated pedicle-screw fixation in low bone quality patients: a case series and literature review. $J$ Biol Regul Homeost Agents. 2018;32(6 suppl 1):71-76.

14. Chandra VVR, Prasad BCM, Jagadeesh MA, Jayachandar V, Kumar SA, Kumar R. Segmental polymethylmethacrylate-augmented fenestrated pedicle screw fixation for lumbar spondylolisthesis in patients with osteoporosis - a case series and review of literature. Neurol India. 2017;65(1):89-95. doi:10. 4103/0028-3886.198229

15. Harrop JS, Styliaras JC, Ooi YC, Radcliff KE, Vaccaro AR, Wu C. Contributing factors to surgical site infections. $J$ Am Acad Orthop Surg. 2012;20(2):94-101. doi:10.5435/JAAOS20-02-094
16. Veeravagu A, Patil CG, Lad SP, Boakye M. Risk factors for postoperative spinal wound infections after spinal decompression and fusion surgeries. Spine. 2009;34(17):1869-1872. doi:10.1097/BRS.0b013e3181adc989

17. Christodoulou E, Chinthakunta S, Reddy D, et al. Axial pullout strength comparison of different screw designs: fenestrated screw, dual outer diameter screw and standard pedicle screw. Scoliosis. 2015;10(1):15. doi:10.1186/s13013-0150039-6

Disclosures and COI: The authors received no funding for this study and report no conflicts on interest.

Corresponding Author: Ankit I. Mehta, MD, FAANS, Director of Spinal Oncology, Assistant Professor and Assistant Program Director, Adjunct Professor of Orthopedic Surgery and Chemical Engineering, Department of Neurosurgery, University of Illinois at Chicago, 912 South Wood Street, 451-N, Chicago, IL 60612. Phone: (312) 355-0510; Fax: (312) 996-9018; Email: ankitm@uic.edu.

Published 26 February 2021

This manuscript is generously published free of charge by ISASS, the International Society for the Advancement of Spine Surgery. Copyright (c) 2021 ISASS. To see more or order reprints or permissions, see http://ijssurgery.com. 